\title{
Preliminary Study of Determining Trash Components in Lint Cottons by Near Infrared Spectroscopy Technique
}

\author{
Liu $\mathbf{Y}^{1^{*}}$, Thibodeaux $\mathrm{D}^{2}$, Foulk $\mathrm{J}^{3}$ and Rodgers $\mathrm{J}^{1}$ \\ 1 USDA, ARS, Cotton Structure \& Quality Research Unit, New Orleans, LA 70124, USA \\ ${ }^{2}$ Fiber Physics LLC, Pickens, SC 29671, USA \\ ${ }^{3}$ FX-Fibers LLC, Clemson, SC 29631, USA
}

"Corresponding author: Liu Y, USDA, ARS, Cotton Structure \& Quality Research Unit, New Orleans, LA 70124, USA. Tel: 504-286-4455; E-mail: yongliang.liu@ars.usda.gov

Rec date: Jul 02, 2014, Acc date: Jul 22, 2014, Pub date: Aug 05, 2014

Copyright: (c) 2014 Liu Y, et al. This is an open-access article distributed under the terms of the Creative Commons Attribution License, which permits unrestricted use, distribution, and reproduction in any medium, provided the original author and source are credited.

\begin{abstract}
The transfer of Near Infrared (NIR) calibration models for the determination of total trash, leaf trash and non-leaf trash components in cotton fibers was conducted between two sets of samples. These samples to be analyzed are inhomogeneous in a bulky state whereas the samples used as calibrations were homogeneous in a ground state. The efficacy of the model transfer was evaluated based on instrumental leaf grade readings of "as is" diverse samples, because current-in-use trash tests cannot generate the trash amount for individual trash components. Results indicated that the predictions from the direct model transfer were unreliable, but they might be acceptable after the correction or conversion of original predictions with standard samples.
\end{abstract}

Keywords: Cotton total trash; Leaf trash; Non-leaf trash; Near infrared spectroscopy; NIR; Model transfer

\section{Introduction}

More automatic machines have been used to pick cotton fibers in the U.S. and other countries, mostly due to the economic factor and the speed [1]. Commonly, the harvested cottons contain some degree of cotton plant-related contaminants and other irregular foreign matter [2,3]. During subsequent ginning and cleaning practices, great efforts have been made to remove these undesired contaminants as much as possible [4]. Excessive processing may lower some end-use qualities of lint fibers (that were acquired during the ginning process to remove the cotton seed), such as the fiber length due to breakage; thus, assessment of cotton trash is important in optimizing the cleaning treatment too.

Trash in commercial cotton bales has been one of several concerns in cotton industry, as it discounts the value of cotton, requires more cleaning, and influences the quality of yarn and fabric. Over the years, the Shirley analyzer (SA) [5], high volume instrument (HVI ${ }^{\mathrm{TM}}$ ) [6], advanced fiber information system (AFIS) [7], micro dust and trash analyzer (MDTA) [8], and FibroLab [9] have been developed to determine cotton trash content in the U.S. and other countries. Generally, these methods only produce the amount of total trash, instead of the content for such individual or targeted cotton plant trash components as leaves (leaf and bract), seed coats, hulls, and stems. In large part, this limitation arises from the instrumental systems that analyze various trashes simultaneously in commercialready cotton bales; in other words, these trash testing methods cannot measure the amount of a specific trash component and identify the type of trash.

To evaluate the possibility of a rapid and low-cost technique that can be used, away from the laboratory, in places such as ginning sites, near infrared (NIR) spectroscopy has been attempted for the quantitative prediction of trash contents represented as $\mathrm{HVI}^{\mathrm{TM}}[10]$ and SA index [11,12], and also individual trash components [13]. In this latest study [13], a set of mixtures with known amounts of both clean lint fibers and individual trash components (leaves, seed coats, hulls, stems, and sand/soil) was prepared physically and then the visible/NIR spectral response was related to corresponding trash contents. The observation revealed the potential of visible/NIR technique in the precise and quantitative determination of total trash, leaf trash, and non-leaf trash components, but it also indicated the difficulty in the prediction of non-leaf trash components such as stem, hull, seed coat, and sand/soil. As a different approach, Fortier et al. [14] reported NIR spectral feature of individual or pure plant parts and further for their identifications.

There are considerable studies to explore the likelihood of predictive model transfer between instruments of the same or different types $[15,16]$. Even though instruments are the same, they do not generate exactly the same wavelength (horizontal axis) or absorbance (vertical axis) response when measuring the identical sample, due to both the aging/changing parts of instruments and the variations of environmental conditions. To maintain the efficiency of transferred calibration models, there are a number of approaches, including more calibration and standard validation samples [15], and also better spectral pretreatments and wavelength selection methods [16,17]. In these earlier studies, concentration or content of targeted compounds were commonly used for reference laboratory values for NIR calibration development. As a comparison, the true reference values of individual trash components in regular cottons were extremely difficult to acquire, mostly due to either current-in-use trash protocols cannot provide such information or it is labor intensive to amass different types and sizes of trash manually. In this study, lint cotton samples with various instrumental leaf grades were used, since the instrumental leaf grade was determined by an equation that utilizes $\mathrm{HVI}^{\mathrm{TM}}$ trash readings of percent area and particle count on a sample's surface [18] and so that the magnitude of instrument leaf grade should 
be proportional to trash level in a sample; unfortunately, present $\mathrm{HVI}^{\mathrm{TM}}$ leaf grade measurement cannot provide the trash type, such as leaf trash and non leaf trash. The accumulated knowledge could be of value as a rapid analytical tool to cotton breeders for cotton variety enhancement and also to cotton ginning engineers for trash-removal cleaning device improvement. The main objective of this study was to investigate the feasibility of NIR spectroscopy in the non-destructive and rapid prediction of total trash, leaf trash, and non-leaf trash components in regular bulky cottons, by transferring the NIR models built from grounded mixtures of lint fiber and trash.

\section{Materials and Methods}

\section{Grounded clean fibers, cotton trashes and their mixtures}

The details on the collection of clean lint fibers and five types of trashes, and the subsequent preparation of their mixtures were described previously [13]. Briefly, clean lint fibers were obtained from routine SA (Shirley Developments, Ltd., Stockport, UK) processing of different lint cotton varieties, whereas each of the five cotton trash components, namely leaves (including bracts), seed coats, hulls, stems, and sand/soil, was collected either from the trash remains (or wastes) of the SA processing of commercial cottons or from the unginned (or seed) cottons manually. Both clean fibers and five trashes were grounded in a Wiley mill and passed through a 20-mesh screen with a sieve hole size of $0.841 \mathrm{~mm}$. Then, 100 mixtures were prepared subjectively in the way of as homogeneous as possible by re-passing the screen a second time. Each mixture weighted $5.0 \mathrm{~g}$ in total and consisted of cut fibers and five trashes at varying concentration in percentage (\%).

\section{Bulky cotton fibers and instrumental leaf grade reading}

Three hundred lint cotton samples from the 2010 crop-year, grown in the U.S. and with instrumental leaf grade assignment of 1 to 6 were utilized. They represented the diversities in Upland cotton varieties, growth locations, and ginning practices within the U.S. These bulky or uncut fibers were measured under a standard conditioning procedure of $65 \pm 2 \%$ relative humidity and $21 \pm 2^{\circ} \mathrm{C}$ temperatures.

\section{Visible/NIR reflectance acquisition}

A Foss XDS rapid content analyzer (Foss NIRSystems Inc., Laurel, MD) was used to acquire visible/NIR reflectance spectra. Approximately $1.3 \mathrm{~g}$ of individual grounded mixture was loaded into a round sample cell with internal dimension of $1 \mathrm{~cm}$-deep x $3.8 \mathrm{~cm}$ diameter. For regular bulky cottons, about $10 \mathrm{~g}$ of fibers was pressed into a Foss coarse granular cell with internal dimension of $3.8 \mathrm{~cm}$-wide x $15.2 \mathrm{~cm}$-long x $4.8 \mathrm{~cm}$-deep, and a $750 \mathrm{~g}$ of extra weight was loaded on the top of fiber samples consistently to keep a good contact between the cotton sample and the optical window. In either module, $\log (1 /$ Reflectance $)$ or $\log (1 / \mathrm{R})$ readings were obtained in the 400 $2500 \mathrm{~nm}$ visible/NIR range at $0.5 \mathrm{~nm}$ intervals. Three spectra, 32 scans per spectrum, were obtained for each sample by repacking, and their mean spectrum was utilized in the analysis.

Actually, the instrumental's optical window of scanning the samples is less than $2.5 \mathrm{~cm}$ in diameter. Unlike the sample was in a stationary module when using a round sampling cell, the sample in granular cell was moved across the optical window and scanned at 8 locations. This led to eight times more scanning surface area using a granular cell than a round cell. Under the experimental setting, it took about $1 \mathrm{~min}$ to scan one sample.

\section{Partial least squares (PLS) models from grounded samples and transfer to bulky fibers}

Two spectral datasets were imported into PLS plus/IQ package in Grams/AI (Version 7.01, Thermo Scientific, Waltham, MA) for PLS regression model development. For individual trash components, 33 (every $3^{\text {rd }}$ sample on the order of the smallest to largest in trash content) spectra were chosen to validate the model that was built from the remaining 67 spectra. In order to compare the prediction models, different combinations of both spectral ranges (e.g., full and narrow regions) and spectral pretreatments (e.g., mean centering (MC), multiplicative scatter correction (MSC), and the first and second derivatives) were attempted. During the protocol, leaving one-sampleout cross-validation method was used, and the suggested optimal factor that generally corresponded to the minimum of the predicted residual error sum of squares (PRESS) was taken. The performance of models was evaluated in the validation set by the parameters of the coefficient of determination $\left(\mathrm{r}^{2}\right)$, Root Mean Square Error of Validation (RMSEV), and Residual Predictive Deviation (RPD) [19]. Next, the models developed from the grounded mixtures were applied to the spectra of bulky samples with various leaf grade assignments.

\section{Results and Discussion}

\section{Development of PLS models from grounded samples}

In an earlier study [13], combinations of full / narrow spectral regions and different spectral pre-processing were utilized to optimize the PLS models for the constituents in grounded samples. Statistics in calibration and validation sets from four spectral regions (405- 2495 $\mathrm{nm}, 405-1095 \mathrm{~nm}, 1105-2495 \mathrm{~nm}$, and 900-1700 nm) were citied in Table 1 for the comparison. In general, the models from a narrow 900-1700 nm region showed the potential of NIR technique for the precise and quantitative determination of total trash and non-leaf trash components, due to their great RPDs. The term "RPD" has been used to evaluate the ability of a spectroscopic model in predicting a property [19], and a value of greater than 3.0 suggests the acceptability of the model for a quantitative implementation.

Despite the fact that leaf trash could be better predicted by the inclusion of 405-750 $\mathrm{nm}$ visible region ( $\mathrm{RPD}>3.5$ ), these is a concern that the natural color of cotton fibers may affect the leaf trash model. This visible range reflects the color information and represents the contributions from the pigmentation compounds present in natural fibers, for example, flavonoids and / or degraded products between a reducing sugar and an amino acid [20,21]. Figure 1 compares normalized spectral response among four clean standard fibers that were used to calibrate $\mathrm{HVI}^{\mathrm{TM}}$ micronaire measurement. Their values of micronaire (determined by both fiber maturity and fineness) were $5.34,4.91,4.00$, and 2.52 , respectively. Larger spectral intensity variation in the $405-750 \mathrm{~nm}$ regions than in other region originates from the subtle difference in $\mathrm{HVI}^{\mathrm{TM}}$ color $+\mathrm{b}$ (yellowness) readings ranging from 15.4 to 17.0 among these samples. Hence, natural color occurrence in cotton fibers could hinder the PLS model transfer efficiency and thus the visible region of $400-750 \mathrm{~nm}$ was excluded in this preliminary study.

Chlorophyll and its degradation derivatives in cotton plant parts significantly contribute to the bands in this visible region, as indicated 
Citation: Liu Y, Thibodeaux D, Foulk J, Rodgers J (2014) Preliminary Study of Determining Trash Components in Lint Cottons by Near Infrared

Page 3 of 5

by the intense band below $750 \mathrm{~nm}$ in the spectra of both leave and non-leave trash (Figure 2). Relative to that of clean cotton fibers, the spectra of leaf and non-leaf trash exhibited the larger log $(1 / \mathrm{R})$ readings in the $400-1000 \mathrm{~nm}$ region and the weaker intensity in the 2020-2200 nm regions. Probably, more chlorophyll (represented by the $675 \mathrm{~nm}$ band) and its degradation derivatives in leaf and non-leaf trashes than in cotton fiber are responsible for intense bands in the 400-1000 $\mathrm{nm}$ region.

\section{Application of PLS models to bulky cotton fibers}

\section{Uncorrected predictions}

Applying the PLS models built from the grounded samples to bulky cotton fibers, unreliable predictions of targeted constitutes were within the line of expectation. For example, predicted total trash and non-leaf trash in cottons with instrumental leaf grade 1 to 6 were negative values
(Figure 3), implying the models could not be applied to unknown samples directly. Nevertheless, as higher leaf grade indicates relative greater trash amount in a sample, it is reasonable to observe an increase of total trash, leaf-trash and non-leaf trash with elevating instrumental leaf grade.

\section{Corrected predictions}

Because of significant difference in sampling grounded or bulky fibers between two cells, resultant spectral difference was observed (Figure 4), in which the round sample cell generate slightly high ranges in absorbance than the granular sample cell. Probably, the sample surface on the round sample cell is not similar to that on the granular sample cell due to differing state of samples that were used for the calibrations and the predictions. Therefore, the predicted values in Figure 3 should be corrected by using the standard or known samples.

\begin{tabular}{|c|c|c|c|c|c|c|c|}
\hline \multirow{3}{*}{ Component } & \multirow{2}{*}{ Range } & \multirow{2}{*}{$\begin{array}{c}\text { Optimal } \\
\text { factor }\end{array}$} & \multicolumn{2}{|c|}{ Calibration Set } & \multicolumn{3}{|c|}{ Validation Set } \\
\cline { 4 - 8 } & & & $\mathbf{R}^{2}$ & $\begin{array}{c}\text { RMSEC } \\
(\mathbf{b})\end{array}$ & $\mathbf{r}^{2}$ & $\begin{array}{c}\text { RMSEV } \\
(\mathbf{b})\end{array}$ & $\begin{array}{c}\text { RPD } \\
\text { (c) }\end{array}$ \\
\hline \multirow{5}{*}{ Total trash } & $405-2495 \mathrm{~nm}$ & 6 & 0.93 & 0.76 & 0.89 & 0.88 & 2.9 \\
\cline { 2 - 8 } & $405-1095 \mathrm{~nm}$ & 6 & 0.93 & 0.75 & 0.92 & 0.75 & 3.4 \\
\cline { 2 - 8 } & $1105-2495 \mathrm{~nm}$ & 6 & 0.93 & 0.76 & 0.93 & 0.75 & 3.4 \\
\cline { 2 - 8 } & $900-1700 \mathrm{~nm}$ & 6 & 0.94 & 0.69 & 0.92 & 0.72 & 3.6 \\
\hline \multirow{5}{*}{ Leaf trash } & $405-2495 \mathrm{~nm}$ & 4 & 0.94 & 0.26 & 0.92 & 0.28 & 3.6 \\
\cline { 2 - 8 } & $405-1095 \mathrm{~nm}$ & 5 & 0.95 & 0.23 & 0.94 & 0.26 & 3.9 \\
\cline { 2 - 8 } & $1105-2495 \mathrm{~nm}$ & 6 & 0.87 & 0.38 & 0.84 & 0.41 & 2.5 \\
\cline { 2 - 8 } & $900-1700 \mathrm{~nm}$ & 8 & 0.89 & 0.35 & 0.89 & 0.35 & 2.9 \\
\hline \multirow{5}{*}{ Non-leaf trash } & $405-2495 \mathrm{~nm}$ & 7 & 0.93 & 0.67 & 0.92 & 0.66 & 3.2 \\
\cline { 2 - 8 } & $405-1095 \mathrm{~nm}$ & 6 & 0.91 & 0.73 & 0.9 & 0.68 & 3.1 \\
\cline { 2 - 8 } & $1105-2495 \mathrm{~nm}$ & 7 & 0.95 & 0.54 & 0.91 & 0.69 & 3 \\
\cline { 2 - 8 } & $900-1700 \mathrm{~nm}$ & 8 & 0.95 & 0.54 & 0.92 & 0.60 & 3.5 \\
\hline
\end{tabular}

Table 1: Statistics in calibration and validation sets [13(a)]

a. Spectral pre-treatment with mean entering (MC) and a Savitzky-Golay first derivative function of two degrees and thirteen points for all models.

b. Root mean square error of calibration (RMSEC) and validation (RMSEV).

c. $\mathrm{RPD}=\mathrm{SD} / \mathrm{RMSEV}$.

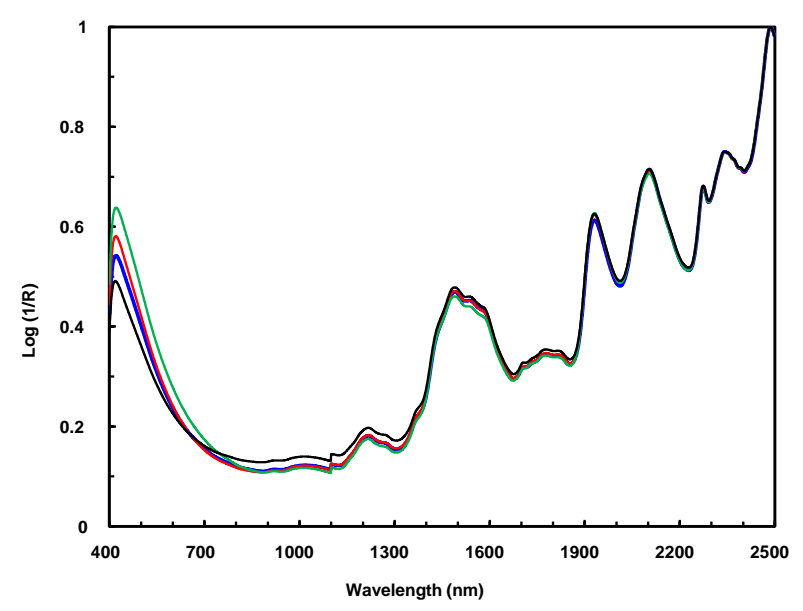

Figure 1: Comparison of normalized visible/NIR log (1/R) spectra of international standard calibration cotton fibers.

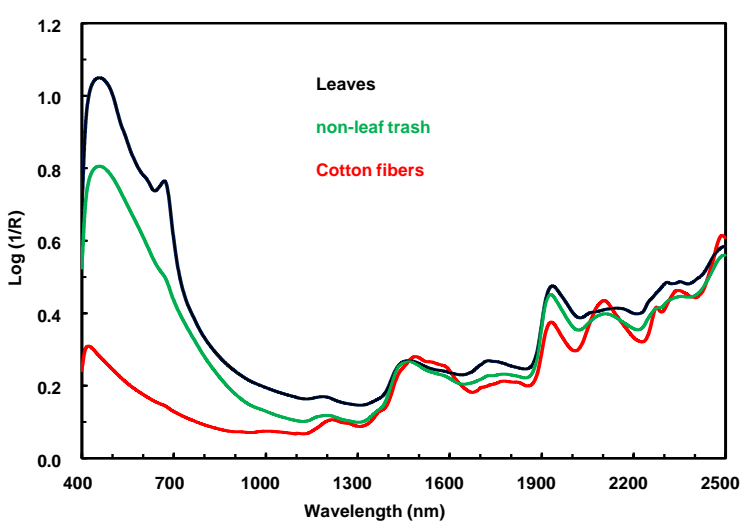

Figure 2: Typical visible/NIR log (1/R) spectra of cut clean "cotton fibers", leaf trash, and non-leave trash that included stem, seed coat and hull trash. 
Additional factors such as sample packing intensity and instrumental fluctuation can impede the model transfer.

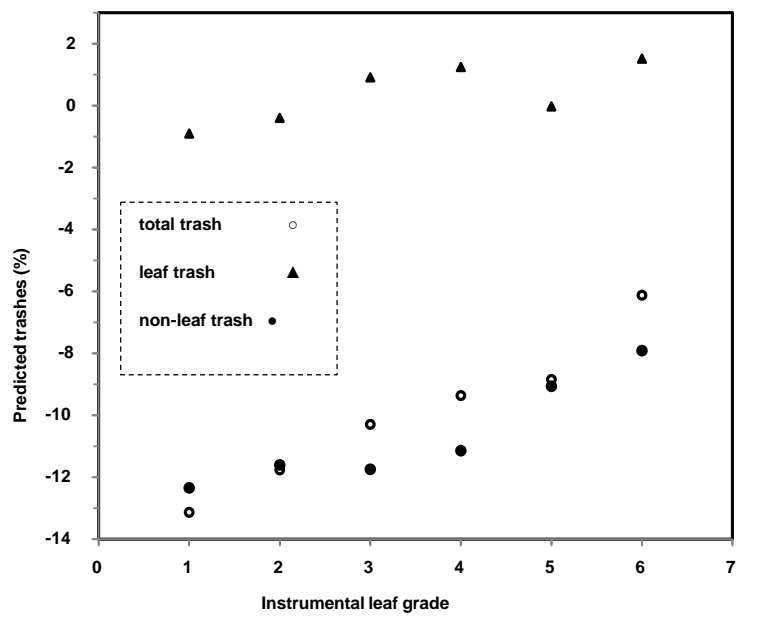

Figure 3: NIR predicted total trash (०), non-leaf trash $(\bullet)$ and leaf trash (ム) from the $900-1700 \mathrm{~nm}$ region. Average of 50 diversified samples was used for each instrumental leaf grade.

In this preliminary study, both one $\mathrm{HVI}^{\mathrm{TM}}$ micronaire standard fiber $($ micronaire value $=5.34$; color $+b=17.0$ ) and pure trash mixture were utilized to correct the predictions in Figure 3. Undoubtedly, the selection of this specific calibration cotton is subject to more discussion and possible impacts of micronaire value and color on model transfer need further investigation. Figure 5 shows the corrected NIR predictions of total trash, non-leaf trash and leaf trash for different instrumental leaf grade samples. These conversions were simply calculated from the following general equation,

$\operatorname{Trash}(\%)=100^{*}(\mathrm{Ps}-\mathrm{Pc}) /(\mathrm{Pt}-\mathrm{Pc})$

where Trash (\%) stands for the corrected content of total trash, or non-leaf trash, or leaf-trash

Through NIR model transfer, Ps, Pc, and Pt are the predicted values on bulky samples, uncut clean cotton fibers, and uncut trash materials from NIR models based on grounded mixtures. Clearly, Pc and Pt change with total trash, non-leaf trash, and leaf-trash variable. In this approach, the clean cotton fiber and trash components are considered to have the trash values of 0.0 and $100 \%$, respectively.

The results in Figure 5 are promising, but undoubtedly it still has the space to improve the predictions in the future. One ultimate question is how to verify the results in Figure 5, in which the validation of model transferability was based on the instrumental leaf grade instead of measured trash contents. As stated earlier [13], trash determination tools can be categorized into two main groups: (1) gravimetric methods that assess the trash content by weight $(\mathrm{g})$ or percentage (\%) and (2) geometric method that estimate the trash content by trash particle count and percentage area on a sample's surface. Because of the heterogeneous distribution of trash type, density, and particle size, it is a challenge to link the trash amount from either type of two trash measurements with NIR spectra at satisfactory level, as revealed by relatively low NIR model performance for referenced SA and HVI ${ }^{\mathrm{TM}}$ trash indices $[11,12]$. As an alternative to overcome the challenge in NIR prediction of trash contents, sevenclass SIMCA/PCA (soft independent modeling of class analogy / principal component analysis) classification models in different visible/NIR spectral regions were developed to optimize the identification efficiency of cotton samples with various leaf grade categories [18]. It was observed that using the discrimination model in the 1105-1700 nm NIR regions could distinguish one class of leaf grade fibers from other six groups at a satisfactory level of $\sim 95.0 \%$.

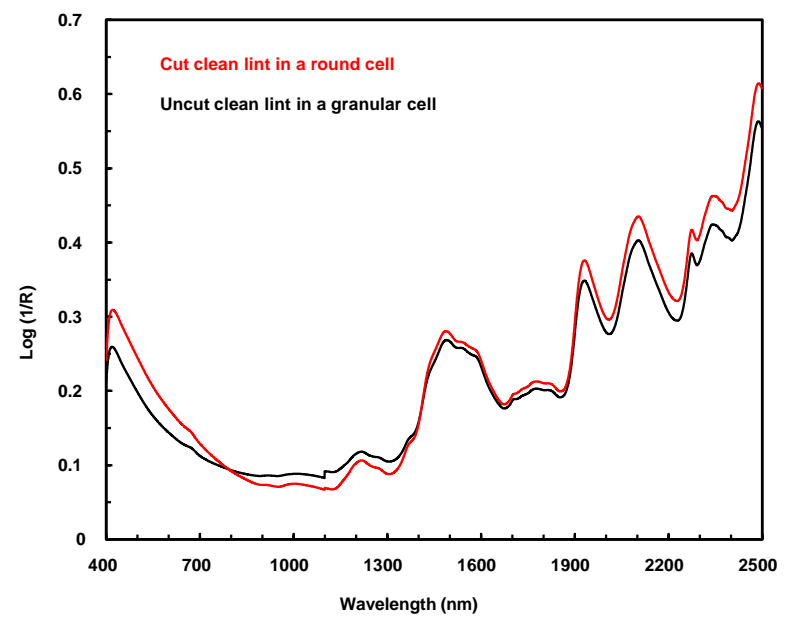

Figure 4: Comparison of visible/NIR log (1/R) spectra of clean cotton fibers between two types of sampling cells.

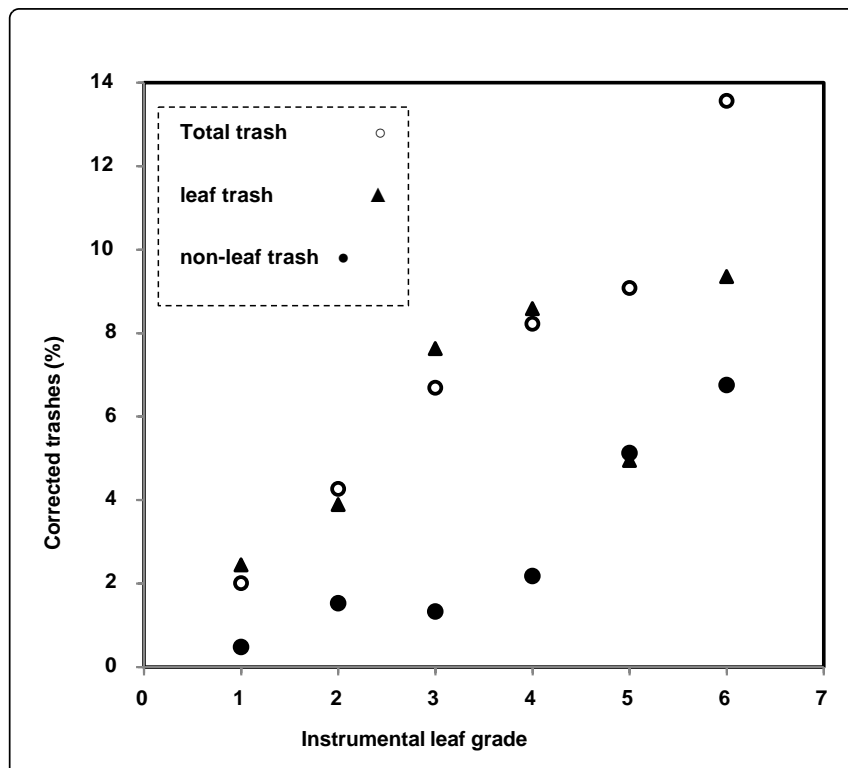

Figure 5: Corrected NIR total trash $(\circ)$, non-leaf trash $(\bullet)$ and leaf trash $(\mathbf{\Lambda})$ for different instrumental leaf grade samples. 
Citation: Liu Y, Thibodeaux D, Foulk J, Rodgers J (2014) Preliminary Study of Determining Trash Components in Lint Cottons by Near Infrared Spectroscopy Technique. J Textile Sci Eng 4: 161. doi:10.4172/2165-8064.1000161

Page 5 of 5

\section{Conclusions}

The transfer of NIR calibration models for the determination of total trash, leaf trash and non-leaf trash components in cotton fibers was attempted. In this scenario, the samples to be analyzed are inhomogeneous in a bulky state whereas the samples used as calibrations were much homogeneous in a ground state. Difference in sampling state (ground vs. bulky) generated a differing spectral response, and reasonably, direct transfer of NIR models built from grounded mixtures to bulky cotton fibers encounter some challenges. With the utilization of standard samples to correct or convert the original predictions, the results implied the feasibility of NIR technique for the rapid and quantitative determination of total trash, leaf trash and non-leaf trash components in commercial cotton fibers.

\section{Acknowledgements}

We sincerely thanked Mr. James Knowlton (USDA, AMS, Memphis, TN) for meeting our request of providing the diversified cotton samples and appreciated the technical assistance from Ms. Mattie Morris (retired from ARS). We also appreciated the useful comments from Leon Cui and Chris Delhom (USDA, ARS, New Orleans, LA).

Mention of a product or specific equipment does not constitute a guarantee or warranty by the U.S. Department of Agriculture and does not imply its approval to the exclusion of other products that may also be suitable

\section{References}

1. Wakelyn PJ, Edwards JV, Bertoniere NR, Triplett BA, Hunter L et al. (2007) Cotton Fiber Chemistry and Technology, CRC Press, Boca Raton, FL, USA.

2. Liu F, Su Z, He X, Zhang C, Chen M et al. (2014) A laser imaging method for machine vision detection of white contaminants in cotton. Text Res J.

3. Delhom CD (2014) Fiber quality challenges facing the cotton industry. Proceedings of the 2014 Beltwide Cotton Conference, 590-592.

4. Gordon S, Hsieh Y-L (2007) Cotton: Science and Technology, Wood head publishing limited, Cambridge, England.

5. American Society for Testing and Materials (ASTM) (2012) Standard test method for non-lint content of cotton, ASTM International, West Conshohocken, PA, USA.
6. American Society for Testing and Materials (ASTM) (2012) Standard test method for measurement of physical properties of cotton fibers by high volume instruments, ASTM International, West Conshohocken, PA, USA.

7. American Society for Testing and Materials (ASTM) (2012) Standard test method for neps in cotton fibers, ASTM International, West Conshohocken, PA, USA.

8. Boykin J, Armijo C, Whitelock D, Buser M, Holt G et al. (2009) Fractionation of foreign matter in ginned lint before and after lint cleaning. Trans ASABE 52: 419-426.

9. Matusiak M, Walawska A (2010) Important aspect of cotton color measurement. Fibers \& Text in Eastern Europe, 18: 17-23.

10. Thomasson JA, Shearer SA (1995) Correlation of NIR data with cotton quality characteristics. Trans ASAE 38: 1005-1010.

11. Liu Y, Gamble G, Thibodeaux D (2010) Evaluation of 3 cotton trash measurements methods by visible/near-infrared reflectance spectroscopy. ASABE, St. Joseph, MI, USA.

12. Liu Y, Gamble G, Thibodeaux D (2010) Assessment of recovered cotton fiber and trash contents in lint cotton waste by UV/visible/near-infrared reflectance spectroscopy. J Near Infrared Spectroscopy 18: 239-246.

13. Liu Y, Thibodeaux D (2013) Feasibility of visible and near infrared spectroscopy in the determination of cotton trash components. J Cotton Sci 17: 195-201.

14. Fortier C, Rodgers J, Cintron M, Cui X, Foulk JA (2011) Identification of cotton and cotton trash components by Fourier transform near-infrared spectroscopy. Text Res J, 81: 230-238.

15. Mark H, Workman J (2013) Calibration transfer. Spectroscopy 28: 24-37.

16. Sohn M, Barton FE, Himmelsbach DS (2007) Transfer of near-infrared calibration model for determining fiber content in flax: effects of transfer samples and standardization procedure. Applied Spectroscopy 61: 414-418.

17. Wang J, Xing Z, Qu J (2013) NIR model transfer based on wavelet transform algorithms. Spectroscopy 28: 36-41.

18. Liu Y, Foulk J (2013) Potential of visible and near infrared spectroscopy in the determination of instrumental leaf grade in lint cottons. Text Res J, 83: 928-936.

19. Ozaki Y, McClure WF, Christy AA (2007) Near-Infrared Spectroscopy in Food Science and Technology, John Wiley \& Sons, Hoboken, NJ, USA.

20. Gamble G (2008) Method for the prediction of the rate of $+b$ color change in upland cotton (Gossypium hirsutum L.) as a function of storage temperatures. J Cotton Sci 12: 171-177.

21. Hua S, Wang X, Yuan S, Shao M, Zhao X et al. (2007) Characterization of pigmentation and cellulose synthesis in colored cotton fibers. Crop Sci, 47: $1540-1546$ 